\title{
Compressive Sampling Using a Pushframe Camera
}

\author{
Stuart Bennett ${ }^{2}$,Yoann Noblet ${ }^{1}$, Paul Griffin ${ }^{1}$, Paul Murray $^{2}$, Stephen Marshall $^{2}$,John Jeffers ${ }^{1}$, Daniel K. L. Oi ${ }^{1}$ \\ ${ }^{I}$ SUPA Department of Physics, University of Strathclyde, 107 Rottenrow, Glasgow G4 ONG, UK \\ ${ }^{2}$ Centre for Signal \& Image Processing, Department of Electronic \& Electrical Engineering, University of Strathclyde, 204 George Street, \\ Glasgow G1 $1 X W$, UK
}

\begin{abstract}
Pushframe parallellized single pixel camera imaging utilizes scanning motion to apply linear sampling masks to rapidly compressively sense a scene. We demonstrate strongly performing static binarized noiselet mask designs, tailored for pushframe hardware. (C) 2021 The Author(s)
\end{abstract}

\section{Introduction}

The 'pushframe' camera concept [1], is a parallelized single pixel camera (SPC). A spatial light modulator (SLM) optically imposes a 2D mask on the incoming image, but rather than concentrating all unmasked light on a single photodiode, the pushframe architecture optically sums the light along one axis only, focusing each masked image column on to a separate photodiode, and hence captures the image as a whole on to a 1D sensor array. If there is relative motion between the camera and the scene, similar to that required by a pushbroom sensor, an appropriately designed 2D mask can remain still whilst an accurate image of the scene is stored, a coefficient being recorded for every scene column at every mask column (Figure 1). The benefit of this imager, relative to a pushbroom sensor, is improved signal to noise ratio, as multiple exposures are combined to form the reconstructed image.

Noblet et al.'s architecture paper [1], introduced a simple sampling scheme, based on a Walsh-Hadamard matrix, using a complete set of linearly independent 1-D patterns achieving good scene reconstruction. However, this requires $\boldsymbol{n}$ samples to reconstruct an $\boldsymbol{n}$ pixel image: while there are SNR advantages, compared to the use of a pushbroom sensor, there are no data storage and transmission improvements. The papers of Candès et al. [2], and Donoho [3], established the field of compressive sampling (CS), where a signal is undersampled in a specific way, but then reconstructed near-perfectly through the use of prior knowledge of the signal's properties — in particular that in some basis the signal has a sparse representation, and the samples have been projected on to a different basis.

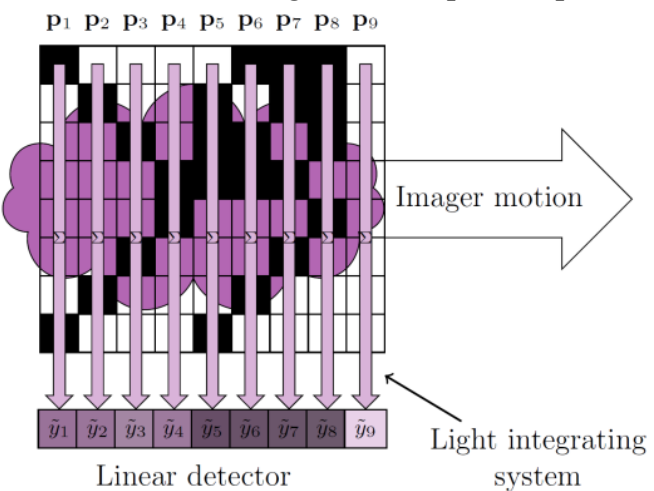

Figure 1: Pushframe capture, column masks modulate scene intensities then summed onto a linear detector array, giving the coefficients used in reconstruction.

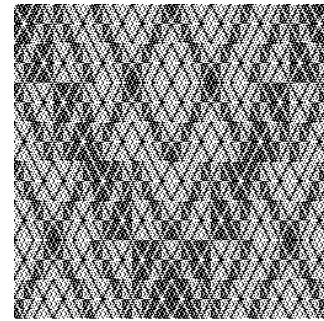

(a)

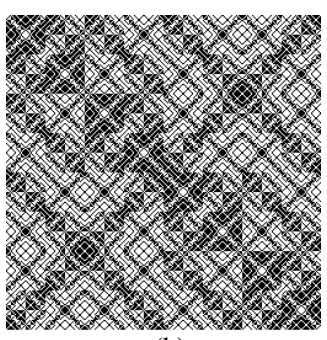

(b)
Figure 2: Alternative SLM Patterns. (a) A full $n=256$ binarized noislet matrix, following Pastuszczak et al.'s mapping, and $(b)$ a full $n=256$ binarized noislet matrix, with mirrored mapping. Empirically, $(b)$ is more optically robust against imperfections in the imaging system.

\section{Methods}

We describe a bespoke pushframe CS scheme. The algorithm design is partially dictated by the combination of pushframe hardware and an Earth-observation application: the rapid scanning motion of the camera over the planet leaves insufficient time to significantly vary the sampling pattern. The SLM hardware, e.g. digital micromirror device (DMD) for broadband capture implements a binary (ON/OFF) mask. The constraint of the sensing matrix to binary values restricts the range of candidate deterministic constructed sensing bases. Pastuszczak et al.'s binary representation of discrete noiselets [4], a family of functions first described by Coifman et al. in [5], which have fast $\mathrm{O}(\mathrm{n} \log \mathrm{n})$ transforms, is ideal for DMD CS imaging (Figure 2). Furthermore, in the reconstruction process we exploit correlations between adjacent columns to recover 2D structure, similar to the process used in $[6,7]$. 
The pushframe architecture elaborated by Noblet et al. [1], can simultaneously capture intrinsically coregistered images in multiple wavelengths, by the use of beamsplitting, diffraction of the integrated light on to an area sensor, or a color (Bayer filtered) camera. Strong spectral correlation between spectral bands can be additionally exploited to further improve multispectral reconstruction of remote sensing images (Figure 3).

\section{Results}

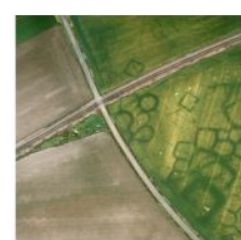

(a) Colou

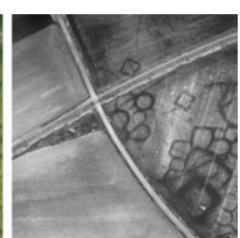

(b) Red

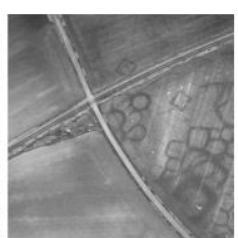

(c) Green

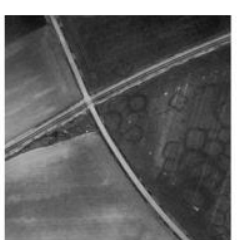

(d) Blue

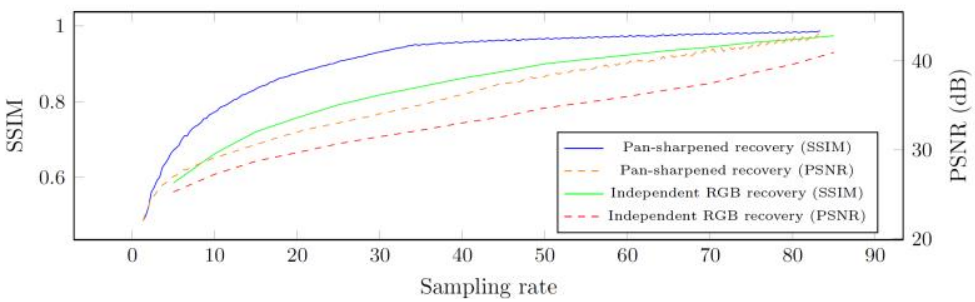

Figure 3: Comparison of image recovery performance using heterogeneous (overall sampling ratio $\underline{m}$ ) and homogeneous $(m)$ band sampling, using pan-sharpening and independent $\mathrm{RGB}$ reconstruction respectively

By using a block-based reconstruction approach, with column-high blocks, our technique delivers good performance, both in simulation and on real-world data, exploiting 2D spatial structure in an imaged scene. It is capable of efficient hardware implementation and it is straightforward to sample with a complete binarized noiselet matrix, and then retain only desired combinations of coefficients for image recovery. We optimize by arranging the sampling basis functions to obtain superior results on optically captured data. Simulations demonstrate that drawing rows from the noiselet basis matrix uniformly when sampling each block gives better results than repeated use of a subset of rows, (or not using blocks at all). Performance increases as blocks become wider, but with marginal gains. Good recovery is robust to lower sampling rates, with the image quality decreasing gracefully as compression increases. Finally, we have outlined a method for obtaining even greater data compression when multi-spectral samples are captured. Though the pan-sharpening recovery technique is not sophisticated, the $40 \%$ data reduction attained is a powerful demonstration of the utility of our enabling pushframe CS approach — without changing the common mask pattern, variation of different bands' compression levels is very computationally efficient.

\section{References}

[1] Yoann Noblet, Stuart Bennett, Paul F. Griffin, Paul Murray, Stephen Marshall, Wojciech Roga, John Jeffers, and Daniel Oi. "Compact multispectral pushframe camera for nanosatellites". Applied Optics, 59(27):8511-8518 (2020).

[2] Emmanuel J. Candès, Justin K. Romberg, and Terence Tao. "Stable signal recovery from incomplete and inaccurate measurements". Communications on Pure and Applied Mathematics, 59(8):1207-1223 (2006).

[3] David L. Donoho. “Compressed sensing”. IEEE Transactions on Information Theory, 52(4):1289-1306 (2006).

[4] Anna Pastuszczak, Bartłomiej Szczygieł, Michał Mikołajczyk, and Rafał Kotynski. "Efficient adaptation of complex-valued noiselet sensing matrices for compressed single-pixel imaging". Applied Optics, 55(19):5141-5148 (2016).

[5] Ronald Coifman, Frank Geshwind, and Yves Meyer. "Noiselets”. Applied and Computational Harmonic Analysis, 10(1):27-44 (2001).

[6] Bing Ouyang, Fraser R. Dalgleish, Frank M. Caimi, Thomas E. Giddings, Walter Britton, Anni K. Vuorenkoski, and Gero Nootz. "Compressive line sensing underwater imaging system". Optical Engineering, 53(5):051409 (2014).

[7] Bing Ouyang, Weilin W. Hou, Frank M. Caimi, Fraser R. Dalgleish, Anni K. Vuorenkoski, and Cuiling Gong. "Integrating dynamic and distributed compressive sensing techniques to enhance image quality of the compressive line sensing system for unmanned aerial vehicles application". Journal of Applied Remote Sensing, 11(3):032407 (2017). 